\title{
Bibliocanta
}

\section{Mapeamento de competências para a implantação e gestão de repositórios institucionais}

Barbosa $^{1}$, Everton Rodrigues evertonpos@gmail.com

Resumo: Aborda o mapeamento de competências para a criação e manutenção de Repositórios Institucionais, a partir da visão do gestor do repositório institucional da Universidade Federal do Rio Grande do Norte. Apresenta um panorama sobre a criação dos RIs no Brasil, e as competências gerais exigidas ao profissional bibliotecário frente a promoção de produtos e serviços de informação em plataformas digitais. Utiliza pesquisa bibliográfica. Analisa qualitativamente os dados de acordo com os pesos atribuídos às habilidades identificadas. Conclui que as competências consideradas de maior relevância se relacionam com a visão e estratégia organizacional. Esse aspecto denota que o profissional deve primeiramente conhecer a visão e missão da Instituição, bem como políticas e normas internas, para implantar e desenvolver o RI local.

Palavras-chave: Gestão por competência. Mapeamento de competências. Repositórios Institucionais. Bibliotecário.

\section{INTRODUÇÃO}

Os repositórios digitais, de maneira geral, foram criados com o objetivo de reunir, preservar, e facilitar o acesso de documentos. Nesse sentido, sua importância e contribuição estão intimamente relacionadas à promoção do acesso e uso da informação.

O advento dessa nova ferramenta tecnológica provocou mudanças no fluxo informacional na medida em que os processos de produção e veiculação da informação passam a se configurar em ambiente digital eletrônico, utilizando maior suporte de tecnologias de informação e comunicação.

Tais mudanças implicam nos custos da produção, nas características dos usuários de informação, e nas competências dos profissionais envolvidos.

\footnotetext{
${ }^{1}$ Mestre em Engenharia de Produção e Bibliotecário da UFRN. Lattes: http://lattes.cnpq.br/4942878021626166
} 


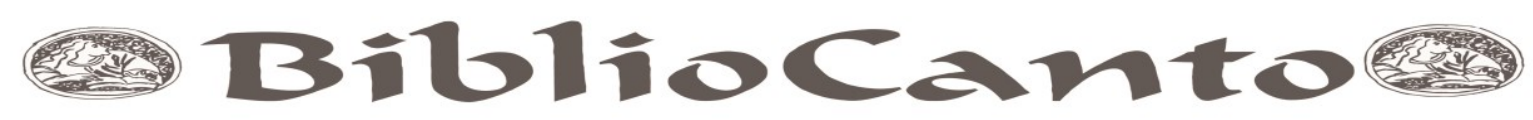

Diante dos avanços tecnológicos e com o intuito de atender as necessidades informacionais de seus usuários, os profissionais da informação devem desenvolver competências profissionais relacionadas ao tratamento da informação em meio eletrônico.

No cenário atual, se destacam os Repositórios Institucionais (RIs) como ferramentas tecnológicas capazes de promover o acesso aberto à informação. Para tanto, a implantação e manutenção dos Rls necessitam de pessoal especializado, com competência para planejar, organizar, controlar e avaliar os recursos digitais, visando a qualidade dos serviços oferecidos.

O cerne da discussão se concentra no levantamento das competências técnicas necessárias para a implementação dos Rls, nas quais estão contextualizadas as habilidades com as tecnologias de informação disponíveis no mercado, bem como a familiaridade com os padrões e técnicas demandadas para disponibilizar e preservar a informação em meio digital. Para isso, deve-se considerar a necessidade de o profissional da informação buscar constantemente se aperfeiçoar.

Nesta perspectiva, pretende-se investigar as competências demandadas aos profissionais que trabalham, especificamente, com os Repositórios Institucionais (Rls), para o perfeito desenvolvimento e manutenção dessas plataformas, considerando as atividades desempenhadas pelos setores dos Rls e o grau de importância de determinadas habilidades. Para a consecução dos objetivos, o estudo fará uma análise com base no mapeamento de competências a partir da visão do coordenador do setor de RI da Biblioteca Central Zila Mamede de Universidade Federal do Rio Grande do Norte.

\section{REPOSITÓRIOS INSTITUCIONAIS}

Os repositórios institucionais surgem com o objetivo primeiro de facilitar o processo da comunicação cientifica, promovendo o acesso livre e uso da produção acadêmica, bem como sua guarda e preservação.

Marcondes e Sayão (2009, p. 9), definem repositórios institucionais como: 


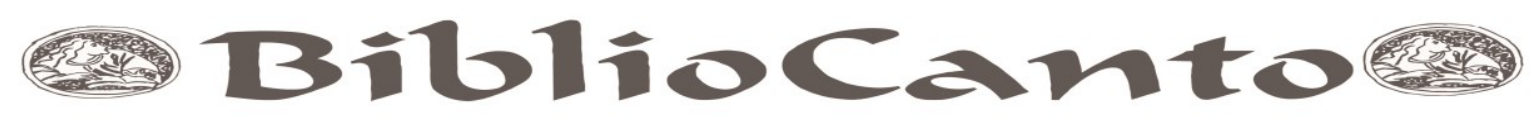

[...] elementos de uma rede ou infra-estrutura informacional de um país ou de um domínio institucional destinados a garantir a guarda, preservação a longo prazo e, fundamentalmente, o livre acesso à produção científica de uma dada instituição. (MARCONDES; SAYÃO, 2009, p. 9).

A principal característica desses repositórios é a política de livre acesso, que segue uma tendência mundial no apoio ao acesso livre da informação cientifica. Esse movimento iniciou a partir de uma reunião de pesquisadores e gestores de repositórios de documentos científicos ocorrida em 1999, denominada Santa Fé Convetion.

O Encontro resultou na criação da Open Archives Initiative (OAI), com a finalidade de "criar mecanismos tecnológicos para tornar interoperáveis os diferentes repositórios funcionando segundo a proposta Open Archives" (MARCONDES; SAYÃO, 2009, p. 15).

Para o desenvolvimento de Repositórios, a OAl estabelece quatro princípios que especificam sua usabilidade e disponibilidade, as quais são: auto arquivamento, interatividade, interdisciplinaridade e interoperabilidade (MACHADO, 2006).

No Brasil, o movimento de acesso livre fundamenta-se no Manifesto Brasileiro de apoio ao acesso livre da Informação Científica, protagonizado pelo Instituto Brasileiro de Informação, Ciência e Tecnologia (IBICT) em 2005.

O IBICT é o órgão nacional, que atua na coordenação dos projetos de Repositórios Institucionais, oferecendo suporte tecnológico para a implantação e manutenção de repositórios nas Instituições de pesquisa e ensino superior do Brasil.

Na UFRN o Repositório Institucional foi implantado em 2008 com o objetivo de reunir a produção intelectual da comunidade universitária (docentes, técnicos e alunos de pós-graduação) da Universidade Federal do Rio Grande do Norte (UFRN), cuja missão é "armazenar, preservar e disponibilizar na Internet, textos completos de acesso livre" (BRASIL, 2010).

A UFRN foi uma das 29 instituições do país a ser contemplado pelo edital do Instituto Brasileiro de Informação e Tecnologia (IBICT) em 2009, e seu pessoal técnico possui conhecimento especializado devido sua experiência de implantação. (MOURA, 2011). 


\section{a Bibliocantos}

O RI-UFRN está amparado em resoluções internas que estabelecem normas sobre a Política Institucional de Informação Técnico-Científica local. Essa considera a necessidade de preservação e ampliação da produção técnico-científica da instituição, bem como potencializar a troca de conhecimentos com outras instituições de ensino e pesquisa. (BRASIL, 2010).

Apesar dos esforços de pesquisadores e gestores dos Rls para divulgar suas experiências, existe ainda uma lacuna no campo das competências técnicas necessárias para a implantação de Repositórios Institucionais. Portanto, para iniciar uma discussão sobre essa temática, faz-se necessário um levantamento das competências do profissional da Informação para a implementação dos Ris.

\subsection{Competências Técnicas para Implementação de Rls}

O principal papel do profissional bibliotecário é trabalhar como mediador, utilizando técnicas para organizar e tratar a informação em função do seu acesso e uso. Nesta perspectiva, suas competências estão relacionadas com o domínio dessas técnicas.

Em busca de atender as necessidades informacionais de seus usuários, considerando o avanço das tecnologias de informação e comunicação, o profissional da informação necessita se aperfeiçoar constantemente, e para isso, sua formação deve estar alinhada as novas tendências.

Nas diretrizes curriculares para os cursos de Biblioteconomia no país, propostas pela Associação Brasileira de Educação em Ciência da Informação (ABECIM, [2000?]), verificam-se disciplinas voltadas para a organização e tratamento da informação, recursos e serviços de informação, fundamentos teóricos da biblioteconomia, e gestão de unidades de informação. Ao longo das últimas duas décadas, cresce a inserção de disciplinas voltadas para administração de unidades de informação e marketing, que conforme Almeida (2012) garantem a qualidade no planejamento, avaliação e promoção de serviços de informação.

Na Classificação Brasileira de Ocupações (CBO), o bibliotecário (profissional da informação) tem como funções: 
Disponibilizar informações para qualquer tipo de suporte; gerenciar unidades como bibliotecas, centros de documentação, centros de informação e correlatos, além de redes e sistemas de informação; tratar tecnicamente e desenvolver recursos informacionais; disseminar informações com o objetivo de facilitar o acesso e geração do conhecimento; desenvolver estudos e pesquisas; realizar a difusão cultural; desenvolver ações educativas; prestar serviços de assessoria e consultoria (CBO, 2013).

Analisando as funções elencadas pela CBO acima, observa-se que se aplicam ao profissional da informação em qualquer campo de atuação, seja em ambientes tradicionais ou digitais.

Nesse contexto, o Draft Guidelines on Cultural Work within the Information Society, do Council For Cultural Co-Operation (2000) recomenda quanto ao uso das tecnologias de informação que os profissionais devem possuir habilidades para dominar as TICs, adotar tecnologias inovadoras, dominar formatos e métodos normalizados para descrição, apresentação e transmissão de dados, bem como de navegar nas redes de comunicação.

No âmbito dos serviços de informação digitais, os Ris vêm se destacando no cenário nacional, e estudos mostram que a grande maioria delas se estabeleceram em Bibliotecas de instituições de ensino e pesquisa, tendo como precursores na implantação os profissionais da informação. De acordo com Leite (2009) isso ocorre, porque as atividades inerentes ao desenvolvimento dos Ris apresentam semelhanças se comparado aos trabalhos desenvolvidos em ambientes digitais por bibliotecas e bibliotecários.

Segundo Leite (2009) para a construção de repositórios institucionais de acesso aberto à informação científica, os profissionais devem considerar um conjunto de instruções específicas que surgem das melhores práticas de implementação, nas quais incluem aspectos como:

a) Planejamento: custos, equipe e competências, análise contextual, definição e planejamento, avaliação das necessidades de comunicação; 


\section{DBibliocantoo}

b) Implementação: Escolha do software, metadados, diretrizes para a criação de comunidades, fluxos, elaboração de políticas de funcionamento, projeto-piloto;

c) Assegurando a participação da comunidade: Marketing e povoamento do repositório, política de depósito compulsório, avaliação e indicadores de desempenho do RI.

Atrelado a esses aspectos tem-se as estratégias para construir o sistema global e aberto de gestão e comunicação do conhecimento científico.

Robinson (2007) elenca uma série de habilidades necessárias às atividades de desenvolvimento e gestão de repositórios, a saber: gestão; avaliação e seleção de softwares apropriados; conhecimento e aplicação de padrões de metadados; melhores práticas e procedimentos para o armazenamento e preservação dos conteúdos; questões relevantes quanto as políticas de proteção de propriedade intelectual e direitos autorais; e o estreitamento dos laços com os atores sociais envolvidos no processo de produção científica, tais como provedores de serviços, sociedades científicas e outras universidades ou institutos de pesquisa que colaboram com as políticas de acesso aberto a informação.

As habilidades relacionadas aos aspectos da gestão são consideradas fundamentais para a implantação e manutenção dos Repositórios Institucionais, tendo em vista a necessidade dos profissionais em desenvolver estratégias e coordenar as atividades das equipe e setores colaboradores, realizar levantamentos de satisfação de usuário, avaliar serviços, elaborar fluxos para o gerenciamento de conteúdo, e identificar objetivos e metas para desenvolver estratégias de melhorias para os serviços digitais oferecidos pelos RIs. (ROBINSON, 2007).

Para Sayão e Marcondes (2009) a gestão do repositório deve compreender a administração das coleções, dos usuários finais e da segurança da informação. Nessa perspectiva, devem ser avaliadas as funcionalidades e ferramentas que apoiem a perfeita implantação e manutenção dos serviços digitais, considerando as ferramentas de controle estatístico, controle de direitos autorais, interface administrativa, preservação digital e gestão do usuário final. 


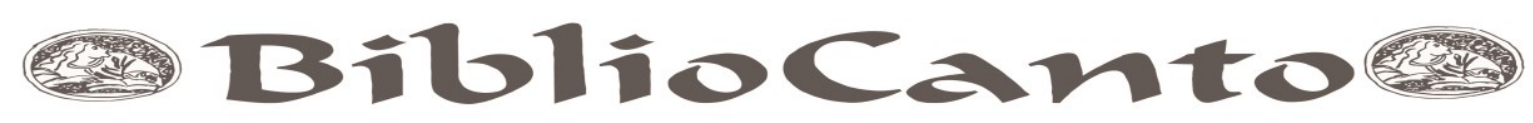

As atividades mencionadas acima correspondem a um levantamento geral de algumas competências técnicas importantes para a promoção de produtos e serviços de informação no ambiente digital. Portanto, é de extrema importância que bibliotecários adequem o seu fazer profissional às ferramentas informacionais que se apoiam nas novas tecnológicas de informação e comunicação.

O levantamento de tais competências possibilita uma visualização geral das habilidades demandadas pelos profissionais da informação na era digital. Porém, para que tais competências sejam consideradas na implementação dos Rls, será preciso desenvolver um estudo direcionado, baseado no mapeamento de competências técnicas especificas que estejam alinhadas com missão e metas organizacionais.

\section{MAPEAMENTO E GESTÃO POR COMPETÊNCIAS}

O termo competência no campo da gestão é definido como a capacidade ou aptidão profissional expresso através de conhecimentos, habilidades e atitudes dentro de um contexto organizacional. (CARBONE et al., 2009; BRANDÃO; GUIMARÃES, 2001).

Desta forma, a competência profissional está intimamente relacionada às habilidades do indivíduo em consonância com as habilidades exigidas pela organização, para a consecução dos objetivos e metas da corporação.

Nessa oportunidade, Brandão e Bahry (2005, p.180) elencam dois tipos de competência:

a) Competências humanas ou profissionais - aquelas relacionadas a indivíduos ou a pequenas equipes de trabalho;

b) Competências organizacionais - aquelas inerentes a toda a organização ou a uma de suas unidades produtivas.

A disciplina encarregada para mapear as habilidades profissionais é a gestão por competência, que surge com o objetivo de orientar esforços para planejar, captar, desenvolver e avaliar, nos diferentes níveis da organização, as 


\section{Dibliocantos}

competências necessárias à consecução de seus objetivos em consonância com a estratégia e missão da organização.

Segundo Brandão e Bahry (2005) esse processo se inicia a partir da formulação da estratégia organizacional, e continua com o levantamento de indicadores de desempenho e metas, se tornando um processo cíclico e contínuo, na medida em que permite fazer o acompanhamento dos processos de trabalho para então oferecer o feedback aos gestores.

O Mapeamento de competências, embora fundamentado na Gestão por competências, busca definir e descrever as capacidades comportamentais ou técnicas necessárias a cada cargo da instituição, permitindo que a organização desenvolva seus critérios de mensuração de sucesso, alinhados às estratégias e missão. Portanto, esse processo se caracteriza como o primeiro passo para a Gestão por Competências. (CARBONE, 2009).

De acordo com Bruno-Faria e Brandão (2003), o mapeamento deve ser feito com pessoas-chave da organização, e a descrição das competências devem se basear em comportamentos ou desempenhos desejáveis, que indicaram como o profissional deve agir diante das situações de trabalho.

Esse levantamento pode ser feito através da aplicação de diversas técnicas, utilizando questionários com perguntas abertas, entrevistas individuais ou coletivas, observação participante ou escala para avaliar o grau de importância de cada competência descrita (MATTAR, 1996; BRUNO-FARIA; BRANDÃO, 2003; CARBONE et al., 2009).

\section{PERCURSO METODOLÓGICO}

O Estudo caracteriza-se como exploratório descritivo, que visa fazer um levantamento bibliográfico sobre o tema, utiliza de entrevista sistemática para então descrever as características de um determinado fenômeno ou população. (GIL, 2007).

Esse estudo não pretende analisar as lacunas existentes entre as habilidades preteríveis e as habilidades existentes na organização, e sim identificar 


\section{a Bibliocantoo}

as competências mais relevantes para a criação dos Rls, caracterizando-se como um primeiro passo para aplicação de métodos da Gestão por Competências.

Para a coleta dos dados, utilizou-se questionário aplicado ao gestor do RI da UFRN, com questões elaboradas e fundamentadas na revisão de literatura, utilizando o método apresentado por Carbone et al. (2009), em que as competências descritas são avaliadas a partir da visão do gestor.

Os dados foram analisados qualitativamente, a partir da análise dos índices de relevância para cada competência descrita, atribuídos pelo gestor do RI-UFRN, observando os seguintes pesos: 1 - Desejável; 2 - Importante; 3 - Essencial.

\section{ANÁLISE DOS RESULTADOS}

A partir da visão e experiência do gestor do Repositório Institucional da UFRN, bem como as demandas identificadas pelo referido setor, as competências técnicas consideradas mais importantes para a implantação e manutenção dos RIs são dispostas no quadro abaixo.

Quadro 1 - Competências técnicas para implantação e manutenção dos Rls

\begin{tabular}{|l|c|}
\hline \multicolumn{1}{|c|}{ Competências técnicas } & PESOS \\
\hline $\begin{array}{l}\text { Conhecimento sobre as Normas da ABNT para a elaboração de trabalhos } \\
\text { acadêmicos }\end{array}$ & 2 \\
\hline $\begin{array}{l}\text { Conhecer regras e padrões no campo da representação descritiva da } \\
\text { Informação }\end{array}$ & 3 \\
\hline Conhecimento sobre Indexação & 2 \\
\hline $\begin{array}{l}\text { Conhecimento aprofundado da Internet, dos seus serviços e } \\
\text { potencialidades }\end{array}$ & 2 \\
\hline Capacidade para criar e gerir conteúdos na World Wide Web (HTML, etc.) & 2 \\
\hline Capacidade de liderar e motivar equipes de trabalho & 2 \\
\hline $\begin{array}{l}\text { Capacidade de divulgar produtos e serviços utilizando diversos } \\
\text { canais de informação }\end{array}$ & 3 \\
\hline Conhecimento de normas e regimentos internos da organização & 3 \\
\hline
\end{tabular}

BiblioCanto, Natal, v. 2, n.1, p. 21 - 34, 2016 


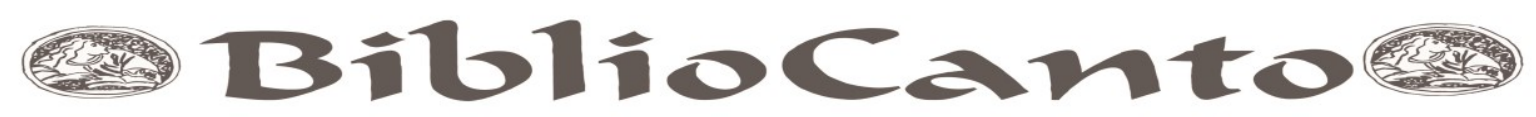

\begin{tabular}{|l|c|}
\hline Capacidade de ler, compreender e se comunicar em outros idiomas & 2 \\
\hline Conhecer ferramentas que viabilizem os estudos métricos da informação & 2 \\
\hline $\begin{array}{l}\text { Conhecimento sobre Políticas relacionadas ao acesso livre da } \\
\text { informação cientifica no Brasil }\end{array}$ & 3 \\
\hline Capacidade para usar e avaliar software e hardware diversos & 2 \\
\hline $\begin{array}{l}\text { Conhecimento dos métodos, técnicas e normas de digitalização e/ou } \\
\text { criação de documentos multimídia e da sua disponibilização para o } \\
\text { público (interfaces, design, etc.) }\end{array}$ & 2 \\
\hline $\begin{array}{l}\text { Conhecimento sobre técnicas de preservação e conservação de } \\
\text { documentos digitais }\end{array}$ & 2 \\
\hline $\begin{array}{l}\text { Capacidade de Planejar, implantar, acompanhar e avaliar produtos e } \\
\text { serviços de informação }\end{array}$ & 2 \\
\hline Conhecer a lei de Direitos autorais & 3 \\
\hline $\begin{array}{l}\text { Conhecimento e capacidade de trabalho com os diversos protocolos de } \\
\text { comunicação e transferência de dados - OAl-PMH, Z39.50, etc. }\end{array}$ & 2 \\
\hline
\end{tabular}

Legenda: 3 Essencial - 2 Importante - 1 Desejável.

Fonte: O Autor (2013)

Analisando o quadro acima, verifica-se que a maioria das competências consideradas essenciais se relaciona coma visão Institucional, estabelecida no conhecimento de leis, normas e regimentos que dão suporte ao seu funcionamento, bem como, apoiam e divulgam amplamente seus serviços na Instituição.

Nesse aspecto, se destaca o conhecimento de normas e regimentos internos, demonstrando que os Rls surgem para atender uma necessidade institucional, fundamentada na preservação e divulgação do conhecimento cientifico produzido localmente. Portanto, o profissional precisa primeiramente conhecer o entorno organizacional, pois esse, fornece suporte ao planejamento e tomada de decisões.

Além disso, se deve observar o ambiente externo, acompanhando a tendência mundial dos Open Archives na medida em que se considera essencial o conhecimento de leis e políticas relacionadas ao acesso livre da informação científica e direito autoral. 


\section{a Bibliocantos}

No que se refere às competências técnicas de conhecimentos específicos, destaca-se apenas a necessidade de conhecer regras e padrões no campo da representação descritiva da informação. As demais competências de conhecimentos específicos, tais como Normas Brasileiras para elaboração de trabalhos acadêmicos e indexação, protocolos, padrões de metadados, e uso apropriado de softwares foram assinalados como competências importantes.

Verifica-se também que, nenhuma das competências técnicas elencadas no estudo, foram consideradas como desejáveis no processo de implantação e manutenção dos RIs.

\section{CONSIDERAÇÕES FINAIS}

A partir da revisão da literatura foi possível identificar as competências necessárias ao profissional da informação, frente às novas tecnologias de informação e comunicação, mais especificamente, em se tratando dos Rls, o estudo identificou as capacidades técnicas exigidas ao profissional que pretende atuar em Repositórios Institucionais.

Através da análise dos dados, se percebe que as competências de maior relevância se relacionam com a visão e estratégia organizacional. Esse aspecto denota que o profissional deve primeiramente conhecer a visão e missão da Instituição, bem como políticas e normas internas, que darão suporte à implantação e desenvolvimento do RI local. Outro aspecto considerado essencial é o conhecimento de Políticas de Acesso Livre no Brasil e leis de direito autoral.

Os resultados da pesquisa revelam que os objetivos propostos foram alcançados, pois através do estudo foi possível identificar - de acordo com a visão do gestor e demandas do setor - as competências consideradas relevantes no processo de implantação e manutenção de Repositórios Institucionais. Portanto, se pode afirmar que o domínio das habilidades apresentadas irá contribuir para a gestão da qualidade e manutenção dos produtos e serviços oferecidos.

Deve-se considerar que o estudo se concentrou no mapeamento de competências, ou seja, na identificação das habilidades demandadas pelos 


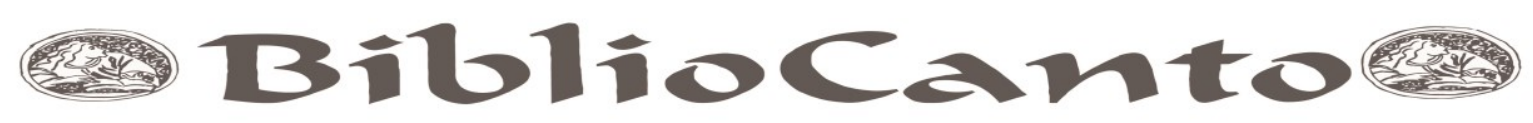

profissionais da informação para manutenção e implementação dos Rls. Desta forma, recomenda-se a elaboração de estudos futuros que objetivem identificar as lacunas entre as capacidades preteríveis e as capacidades existentes, para então aplicar de maneira efetiva e global a gestão por competências.

Competency mapping for the implementation and management of institutional repositories

\begin{abstract}
This study discusses about competence mapping to institutional repositories management, based on the view of Repositório Institucional da UFRN's manager. It presents a panorama about the Institutional repositories foundation in Brazil, and the librarian competences needed to promote information in digital platforms. It utilizes the bibliographic and electronic research, and analyses this case in qualitative form. It concludes that the main competences are related to the strategy and organizational vision, and the librarian needs to know these aspects to develop the local institutional repositories.
\end{abstract}

Keywords: Competence-based management. Competence mapping. Institutional Repositories. Librarian.

\title{
REFERÊNCIAS
}

ALMEIDA, Neilia Barros Ferreira de. Biblioteconomia no Brasil: análise dos fatos históricos da criação e do desenvolvimento e do ensino. Brasília, DF, 2012.

Dissertação (Mestrado) - Programa de Pós-Graduação em Ciência da Informação, Faculdade de Ciência da Informação, Universidade de Brasília. Disponível em: <http://repositorio.unb.br/bitstream/10482/11170/1/2012_NeiliaBarrosFerreiradeAlme ida.pdf>. Acesso em 17 jul. 2013.

ASSOCIAÇÃO BRASILEIRA DE EDUCAÇÃO EM CIÊNCIA DA INFORMAÇÃO (ABECIN). Proposta de diretrizes curriculares para a área de Ciência da Informação: Cursos de Arquivologia, Biblioteconomia e Museologia - segunda versão. [2000?]. Disponível

em:<http://www.abecin.org.br/abecin_conteudo.php?id=14>. Acesso em: 17 set. 2016. 
BRANDÃO, Hugo Pena. GUIMARÃES, Tomás de Aquino. Gestão de competências e gestão de desempenho. Revista RAE, FGV, v.41, n.1, jan./mar. 2001.Disponível em: <http://www.scielo.br/pdf/rae/v41n1/v41n1a02>. Acesso em: 17 jul. 2013.

BRANDÃO, Hugo Pena. BAHRY, Carla Patrícia. Gestão por competências: métodos e técnicas para mapeamento de competências

Revista do Serviço Público Brasília, v. 56, n. 2, p.179-194, abr./jun. 2005.

BRASIL. Ministério da Educação. Universidade Federal do Rio Grande do Norte. Resolução no. 059/2010-CONSEP, 13 de abril de 2010. Natal, RN, 2010. Disponível em:

<http://repositorio.ufrn.br:8080/jspui/sobre/resolucao_592010_consepe_riufrn.pdf> Acesso em: 23 jul. 2013.

BRASIL. Ministério do trabalho e emprego. Classificação Brasileira de Ocupações. Disponível em:

<http://www.mtecbo.gov.br/cbosite/pages/pesquisas/BuscaPorTituloResultado.jsf > . Acesso em: 23 jul. 2013.

BRUNO-FARIA, Maria de Fátima; BRANDÃO, Hugo Pena. Gestão de competências: identificação de competências relevantes a profissionais da área de T\&D de uma organização pública do Distrito Federal. Revista de Administração

Contemporânea, Rio de Janeiro, v. 7, n. 3, p. 35-56, jul./set. 2003. Disponível em: <http://www.scielo.br/pdf/rac/v7n3/v7n3a03>. Acesso em: 24 jul. 2013.

CARBONE, Pedro Paulo et al. Gestão por competências e gestão do conhecimento. 3. ed. Rio de Janeiro: FVG, 2009.

COUNCIL FOR CULTURAL CO-OPERATION. Culture Committee. New Information Technologies" Project: Training, Qualifications and New Professional Profiles: Draft Guidelines on Cultural Work within the Information. Strasbourg, September, 2000.

GIL, Antônio Carlos. Métodos e técnicas de pesquisa social. 5. ed. São Paulo: Atlas, 2007.

LEITE, F. C. L. Como gerenciar e ampliar a visibilidade da informação cientifica brasileira: repositórios institucionais de acesso aberto. Brasília: IBICT, 2009. Disponível em: <http://eprints.rclis.org/13776/1/RI_-_Fernando_Leite.pdf> Acesso em: 23 jul. 2003.

MACHADO, Murilo Milton. Open Archives: panorama dos repositórios.

SantaCatarina, 2006. Dissertação de Mestrado. Programa de Pós-Graduação em Ciência da Informação, Universidade Federal de Santa Catarina. Disponível em: $<$ https://repositorio.ufsc.br/bitstream/handle/123456789/89170/228274.pdf?sequence $=1>$. Acesso em: 23 jul. 2013. 


\section{DBibliocantos}

MARCONDES, Carlos Henrique; SAYÃO, Luis Fernando. Introdução: repositórios institucionais e livre acesso. In: SAYÃO, Luis Fernando. (Org.). Implantação e gestão de repositórios institucionais: políticas, memória, livre acesso e preservação. Salvador: EDUFBA, 2009.p. 9-21.

MATTAR, Fauze Najib. Pesquisa de marketing. São Paulo: Atlas, c1996.

MOURA, Elisângela Alves. Repositório Institucional da UFRN: uma experiência em curso. In: CONGRESSO BRASILEIRO DE BIBLIOTECONOMIA, DOCUMENTAÇÃO E CIÊNCIA DA INFORMAÇÃO, XXIV, 2011, Alagoas. Anais eletrônicos... Alagoas, Maceió, 2011. Disponível em:

<http://febab.org.br/congressos/index.php/cbbd/xxiv/paper/view/541/701>Acesso em: 23 jul. 2013.

ROBINSON, M. Institutional repositories: staff and skills requirements.

SHERPA Document. 2007. Disponível em:

$<$ http://www.sherpa.ac.uk/documents/sherpaplusdocs/notts-

Repository\%20Staff\%20and\%20Skills.pdf >. Acesso em: 18 set. 2016.

SAYÃO, Luis Fernando; MARCONDES, Carlos Henrique. Software livres para repositórios institucionais: alguns subsídios para a seleção. In: SAYÃO, Luis Fernando. (Org.). Implantação e gestão de repositórios institucionais: políticas, memória, livre acesso e preservação. Salvador: EDUFBA, 2009.p. 9-21. 